\title{
Quantitative image analysis to characterize the dynamics of Listeria monocytogenes biofilms
}

\author{
M. Mosquera-Fernández ${ }^{\mathrm{a}, \mathrm{b}}$, P. Sanchez-Vizuete ${ }^{\mathrm{c}}$, R. Briandet $^{\mathrm{c}}$, M.L.Cabo $^{\mathrm{a}}$ and E. Balsa- Canto ${ }^{\mathrm{a}}$ \\ ${ }^{a}$ Instituto de Investigaciones Marinas (CSIC), C/Eduardo Cabello 6, E-36208, Vigo (Spain). \\ ${ }^{\mathrm{b}}$ Universidad de Vigo (UVIGO), C/Lagoas Marcosende E-36208, Vigo (Spain). \\ ${ }^{\mathrm{c}}$ INRA, UMR 1319 Micalis, 1 Ave. de la Republique, F-91300, Massy (France). \\ *Corresponding author: ebalsa@iim.csic.es.
}

\begin{abstract}
This work shows that the combination of two-dimensional (2D) and three-dimensional (3D) analyses of images acquired by confocal laser scanning microscopy facilitates the quantitative spatiotemporal characterization of architectures formed by Listeria monocytogenes biofilms. In particular, the analysis of structural features such as maximum thickness, biovolume, areal porosity and maximum diffusion distance allowed elucidating differences in biofilm formation of three L. monocytogenes strains (L1A1, CECT5873 and CECT4032).
\end{abstract}

The analysis showed a common sequence for all strains. In the first phase, independent clusters evolve to interconnected clusters and honeycomb-like structures. Flat biofilms characterized the second phase. The structures disappear in the third phase.

Nevertheless, the duration of the phases differed from strain to strain. L1A1 strain exhibited the slowest dynamics and the thickest biofilms while the strain CECT4032 presented the faster dynamics and the thinnest biofilms. Also, the number of dead cells varies significantly from strain to strain. 
From the results of the analysis, it can be concluded that $2 \mathrm{D}$ parameters are critical to differentiating morphological features while 3D parameters ease the interpretation and comparative study of the different phases during the life cycle of biofilms.

Keywords: Listeria monocytogenes, biofilm structure; confocal laser scanning microscopy; image analysis

\section{INTRODUCTION}

Listeria monocytogenes is a major concern in the food industry. In fact, the last European Food Safety Authority report (EFSA and ECDC, 2015) still indicates a substantial number of food-related listeriosis outbreaks.

Food contamination by L. monocytogenes frequently occurs during post-processing through crosscontamination, in many cases by food contact with unhygienic work surfaces and facilities (Wilks et al., 2006). Although food equipment and contact surfaces are routinely cleaned and disinfected, $L$. monocytogenes can grow and persist in harbourage sites (Carpentier and Cerf, 2011; Lundén et al., 2000). In those sites L. monocytogenes can form biofilms that in turn can be linked to the intrinsic protective character of bacterial communities to disinfectants (Pan et al., 2006; Robbins et al., 2005; Somers and Wong, 2004).

Biofilms are heterogeneous and commonly multispecies, communities embedded in self-produced organic polymeric matrixes that present complex three-dimensional architectures (Characklis et al., 1990; Costerton et al., 1987). This preference to live in biofilms responds to convenient multifactorial resistance to both unfavourable environment and antimicrobial agents (Fux et al., 2005; Hoiby et. al., 2010).

Biofilm structure determines the main physiological processes related to persistence and resistance. For example, diffusion of antimicrobial agents is conditioned by the shape and thickness of biofilms, thus limiting their efficacy towards biofilm elimination. Biofilms that are ten cells thick exhibit diffusion time 100 times longer than those formed by a single layer of cells (Stewart, 2003). 
The complexity of biofilm architectures has motivated the development of an array of microscopic techniques to study them. For instance, atomic forces microscopy (AFM), wide-field fluorescence microscopy (WFM) and scanning electron microscopy (SEM) provide substantial details for the research of biofilms structures (Djordjevic et al., 2002; Marsh et al., 2003; Mosquera-Fernández et al., 2014). Besides, confocal laser scanner microscopy (CLSM) enables in situ and in vivo three-dimensional biofilm optical imaging (Stoodley et al., 1999).

Despite the evident interest of observing the structures or their development, it is also necessary to quantitatively characterise biofilms. In this respect, CLSM together with quantitative image analysis allows for the automatic determination of biofilms thickness, biovolume or roughness. This approach allows for a quantitative comparison of biofilms among different strains, species or environmental conditions (media, temperature, type of culture).

Substantial efforts have been devoted to develop software tools for image analysis. COMSTAT (Heydorn et al., 2000), ISA (Yang et al., 2000), ISA 3D (Beyenal et al. 2004), PHLIP (Mueller et al., 2006) or BIOFILMDIVER (Mosquera-Fernández et al., 2014) have been already used for the purpose of studies related to biofilms.

This contribution focuses on the characterization of the structure and dynamics of L. monocytogenes biofilms. Previous works focused on spatial characterization. Rieu et al., 2008 presented a comparison of biovolume and mean thickness for L. monocytogenes biofilms under batch and flow conditions using CLSM and PHLIP. Bridier et al., 2010 quantified maximum thickness, biovolume and roughness using PHLIP for biofilms formed by 10 L. monocytogenes strains at $24 \mathrm{~h}$. Similarly Guilbaud et al., 2015 deciphered the structural diversity of biofilms formed by 96 L. monocytogenes strains at $48 \mathrm{~h}$ using also mean thickness, biovolume and roughness. These previous works focused their attention on the spatial characterization of biofilms at particular given times. 
In what respects to structure formation and dynamics, the quantitative analysis is still in its infancy. Mosquera-Fernández et al., 2014 used fluorescence microscopy together with 2D image analysis to characterize the spatiotemporal behavior of three L. monocytogenes strains.

This work goes a step further and presents a complete structure characterization covering the whole life cycle of L. monocytogenes biofilms. For this purpose, we combined CLSM with advanced image analysis including two-dimensional parameters critical to differentiate morphological features and threedimensional parameters to facilitate the comparative temporal analysis.

\section{MATERIALS AND METHODS}

\subsection{Bacterial strains and culture conditions}

Bacteria tested were three L. monocytogenes strains related to food industry. CECT4032 (from soft cheese, serotype 4b and lineage I) and CECT5878 (from guinea-pig, serotype 1/2a and lineage II) were obtained from the Spanish Type Culture Collection (Microbial Resource Centre, University of Valencia, Spain). L1A1 (serotype 1/2a/3a, lineage II) was isolated from thermal gloves used in the fishing industry by the Microbiology and Marine Technology Products research group at our institute.

Bacterial stock cultures were kept at $-80^{\circ} \mathrm{C}$ in tryptone soy broth, TSB (BD Difco, USA), containing $50 \%$ glycerol in the ratio 1:1 (v/v). All strains were grown in two consecutive subcultures in TSB medium for $8 \mathrm{~h}$ and $16 \mathrm{~h}$ at $37^{\circ} \mathrm{C}$ (optimal growth temperature).

\subsection{Biofilm formation and fluorescent labeling}

$50 \mu \mathrm{l}$ of an overnight subculture of each strain were inoculated to $200 \mu \mathrm{l}$ of fresh TSB in each well of polystyrene of a 96-well microtiter plate (Greiner Bio-one, France). The subculture had been adjusted to an OD700 $\mathrm{nm}$ of $0.1( \pm 0.001)$ which corresponds to about $10^{8} \mathrm{CFU} / \mathrm{ml}$ according to a previous calibration. 
Microplates were incubated at $25^{\circ} \mathrm{C}$ under static conditions. After $1 \mathrm{~h}$, the total volume $(250 \mu \mathrm{l})$ was removed and replaced by $200 \mu \mathrm{l}$ of TSB to remove from the system the non-adhered bacteria. Biofilm harvesting was performed eight times per strain at $1 \mathrm{~h}, 4 \mathrm{~h}, 24 \mathrm{~h}$ and every $24 \mathrm{~h}$ up to $120 \mathrm{~h}$.

Prior to visualization under the CLSM, $50 \mu \mathrm{l}$ of TSB mixed with $5 \mu \mathrm{M}$ of each stain of FilmeTracer LIVE/DEAD Biofilm Viability kit (Invitrogen, USA), were added to the previous $200 \mu \mathrm{l}$ of TSB. The kit contains two different fluorescent nucleic acid markers, SYTO® 9 and propidium iodide (PI). SYTO® 9 fluorochrome penetrates in all cells while PI only penetrates in damaged cells. The fluorochromes generate a bicolor labeling where the viable cells are depicted in green and non-viable cells in red. The microtiter plates were incubated at $25^{\circ} \mathrm{C}$ in dark conditions for 20 minutes before visualization to enhance labeling.

\subsection{Image acquisition by Confocal Laser Scanning Microscopy (CLSM)}

Images acquisition was performed using the high throughput method described by Bridier et al., 2010. This method relies on the use of microtiter plates with a $\mu$ clear® base that allows a high-resolution imaging of contained biofilms. For this purpose, Leica SP2 AOBS confocal laser scanner microscope (Leica Microsystems, France) was used. Scans were obtained at $400 \mathrm{~Hz}$ using 63x0.8 NA oil immersion objective with a $488 \mathrm{~nm}$ argon laser set at $25 \%$ of intensity. Dual emissions were recorded within the range 500-600 nm to capture red and green fluorescence. One horizontal cross-section of biofilm, corresponding to a real biofilm area of $238 \times 238 \mu \mathrm{m}^{2}$, defines one image or slice. To cover the whole biofilm height of one randomly chosen $(\mathrm{x}, \mathrm{y})$ coordinate, horizontal cross-sections were acquired consecutively along z-axis using a scanning step size of $1 \mu \mathrm{m}$, defining the so-called stacks. Eight stacks obtained at diverse locations inside the biofilms were randomly chosen for the subsequent image analysis. Images were acquired for three biofilm replicas per sampling time and strain.

\subsection{Image qualitative analysis}

IMARIS software enables visual inspection of the images at different times and heights (see illustrative examples in Figure 1). The slice 2D view mode shows one horizontal cross-section at a certain z-axis height that belongs to a stack of images (illustrative examples can found in the supplementary info 
Figures S1 and S2). This mode allows the user to isolate any image of interest and follow the structural evolution using the scroll bar. Each slice can then be processed with the help of BIOFILMDIVER to obtain 2D structural parameters. The blend 3D view mode provides a fixed aerial view playing with the light and transparency to offer a shading effect that aids in the recognition of biofilm structures as e.g. thickness, shape and distribution of cells on XY plane. The section view mode lets the user inspect the environment of any defined portion of the data set (several slices together) displaying three different areas: YX, ZX and ZY, which allows the observation of live and dead cells distributions in the biofilm and get some idea of the population density. The gallery view mode displays all slices of data set as a continuous series of images that allows the user to observe how the structure evolve along the $\mathrm{z}$-axis and when this disappear.

\subsection{Image quantitative analysis}

For the purpose of quantification, three-dimensional and two-dimensional parameters were selected among the most commonly used to characterize biofilms (Bridier et al., 2010; Mosquera-Fernandez et al. 2014; Rieu et al., 2008; Yang et al., 2000). Figure 2 illustrates the steps accomplished for the quantitative image analysis.

The maximum thickness (MxT) of formed biofilms was obtained by means of IMARIS software. MxT values were computed for the eight replicas at each sampling time (up to $120 \mathrm{~h}$ ) when cells were organized forming a clearly differentiable biofilm. The biovolume (BV) was obtained using the software tool PHLIP (Mueller et al., 2006). The biovolume corresponds to the volume occupied by cells. It is calculated by multiplying the number of foreground pixels in an image stack by the voxel volume, being the voxel volume defined as the product of the pixel area and the scanning step size in the z-axis $(1 \mu \mathrm{m}$ in our case) (Kuehn et al., 1998). The mean values of the maximum thickness and the biovolume are plotted against time so as to ease the comparison between different strains. The $75 \%$ confidence regions are also shown (the maximum variability can be observed in Figures S3 and S4 in the supplementary info).

The areal parameters, areal porosity (AP) and maximum diffusion distance (MDD), were obtained by using the software BIOFILMDIVER (Mosquera-Fernández et al., 2014). Every slice in a stack was 
considered individually, and the 2D parameters are computed for the two color channels (GCh, green channel, corresponding to live cells and RCh, red channel, corresponding to death cells) separately. The areal porosity reflects the area occupied by cells in every slice and corresponds to the ratio between the number of void pixels and the total number of pixels. The maximum diffusion distance reflects the maximum distance covered by cells, and it is calculated using the Euclidean Distance Mapping algorithm (Danielson, 1980). The areal porosity and maximum diffusion distance are plotted against time and z-axis position to ease the characterization of the structure.

\section{RESULTS}

\subsection{Image qualitative analysis}

Visual inspection of the biofilms formed by the L. monocytogenes strains, L1A1, CECT5873 and CECT4032 using the IMARIS blend view mode (see Figure 3) revealed that biofilm formation follows several phases. For the case of L1A1, it can be clearly seen that biofilms are persistently growing up until the final time when the number of damaged cells increases significantly and the structure is lost. The images at earlier times ( $24 \mathrm{~h}$ and $48 \mathrm{~h}$ ) show the presence of small clusters (see, Figures 3 and S1 and S2 in the supplementary info). These clusters eventually joined to each other in a honeycomb pattern (see Figure 3 and Figures S1 A3, A4, B4, B5, in the supplementary info). Later this pattern disappears to form an unstructured mass (see Figures 3, S1 and S2). For the case of CECT5873, the phases are similar to those observed for L1A1. However, the relative durations are different. Besides, clusters are notably

larger and damage cells appear earlier in life cycle (Figure 3). Finally, CECT4032 biofilms evolve differently. The honeycomb phase is either too short or missing and cells die much earlier.

\subsection{Image quantitative analysis}

\subsubsection{Three-dimensional parameters}

The analysis of three-dimensional parameters, i.e. MxT and BV, provide further information about the differences among the strains. Maximum height values revealed some differences both in the maximum height and the time this maximum was achieved (Figure 4A). L1A1 produced the highest biofilm with a 
median value of around $50 \mu \mathrm{m}$. The detachment of L1A1 cells started after $96 \mathrm{~h}$. CECT5873 achieved a maximum height of around $30 \mu \mathrm{m}$ at $24 \mathrm{~h}$ and maintained that height during three days after which a fast decay was observed. Remarkably, CECT4032 attained a maximum height of around $25 \mu \mathrm{m}$ later at $72 \mathrm{~h}$ and started a smooth decay right after.

In some sampling times, results showed substantial relative variability (Figure 3A). The maximum relative variability values corresponded to a 16\%, for L1A1 at $96 \mathrm{~h} ; 27 \%$, for CECT5873 at $48 \mathrm{~h}$ and 19\%, for CECT4032 at $48 \mathrm{~h}$. CECT5873 measurements showed a higher variability; this may be explained taking into account the substantial heterogeneity of the biofilms, possibly due to the presence of clusters and deeper emptier areas. In addition, this may indicate that CECT5873 biofilms are certainly more unstable than those formed by L1A1 and CECT4032.

Biovolume confirmed critical differences between L1A1 and the other two strains. Contrary to what we observed in the temporal evolution of MxT, the BV presents two peaks with maximum biovolume of around 5.5 at 48 and $96 \mathrm{~h}$ (Figure 4B). These two peaks may indicate a period of detachment between $48 \mathrm{~h}$ and $96 \mathrm{~h}$. Biofilms are quite packed at $48 \mathrm{~h}$ with small clusters, not yet too high. At $72 \mathrm{~h}$, after a possible period of detachment, the same height is obtained, while the biovolume has decreased, possibly due to cells in the bulk. At 96 h cells have attached again. Therefore, both height and biovolume increased. Right after, cells detach leading to a substantial height reduction. The temporal evolution of BV profiles obtained from CECT5873 and CECT4032 biofilms study were similar to each other (Figure 4B), showing a first fast growth followed by a flat area that lasts 3-4 days and a final decay. Again, relative variability for CECT5873 was quite significant at $48 \mathrm{~h}$.

\subsubsection{Two-dimensional parameters}

The areal porosity of the biofilms was calculated for the different slices and channels. Figure 5 shows the AP profiles throughout time and height for the three strains. For the case of L1A1 GCh profile showed, as expected, that biofilm AP decreases with time up to 96 to grow again until $120 \mathrm{~h}$, and the biofilms were somehow wrinkled (AP presents peaks in the same z-axis value). It should be noted that AP=1 values regard those times and z-values for which biofilm has been either not formed (earlier times) or already 
disappeared (later times). Regarding the RCh plot, it can be clearly seen that cells started dying later as compared to the other strains, and the cells in most profound layers were the ones that started dying the earliest. At $120 \mathrm{~h}$ however, the presence of dead cells was quite significant through all biofilm.

CECT5873 resulted in the minimum AP values as compared to other biofilms. This small AP indicates that this strain tends to cover the coupon, while not focusing on piling in the z-axis. Surprisingly death cells contributed to the maximum height observed at $48 \mathrm{~h}$ (and thus to the variability we found in MxT and BV). Differently to what we observed for L1A1, death cells could be found at any height in the biofilm. This behavior was even more prominent in CECT4032, for which death cells appear to be thoroughly mixed with live cells at all times and heights.

Maximum diffusion distance was evaluated for all slices and channels. Figure 6 presents the results. CECT5873 green channel analysis resulted in the maximum values for MDD, while L1A1 resulted in the lowest MDD values throughout time and height. These results indicate that CECT5873 tends to form larger clusters (at $24 \mathrm{~h}$ ) or honeycomb like patterns $(72 \mathrm{~h}$ ) while L1A1 is wrinkled with a higher number of smaller aggrupations. Of course, the parameter does not provide information about the number or the real size of clusters. Nevertheless the timing and shape of the most relevant clusters can be clearly recovered from the figures. For example, for CECT5873, it can be clearly seen that a mushroom-type cluster appeared at $24 \mathrm{~h}$ with a maximum height of around $30 \mu \mathrm{m}$ (see Figure 6C-6D). CECT4032 formed clusters but not mushrooms, i.e. similar MDD values are obtained at different heights. Concerning dead cells (RGh), for the case of L1A1, cells died at a lower height, with an even distribution, whereas for the other strains, cells appeared to die inside or on the surface of the clusters (Figure 6D\&F).

\section{DISCUSSION}

Qualitative inspection of CLSM images and IMARIS reconstructions together with the 2D and 3D quantitative characterization of Listeria biofilms revealed that biofilm formation is temporally complex as well as spatially complex. The three-dimensional analysis enables to compare maximum height and biovolume among strains. However, 3D parameters do not provide quantitative information about other structural properties such as the presence of clusters or the location of dead cells. In this respect, the 
addition of 2D parameters has shown to be essential to decipher spatial structural events and the location and times of appearance of dead cells in the biofilms.

The limitations of 3D parameters confirm what it had been discussed in the literature on the necessity of combining different parameters to achieve a better knowledge about the structure of biofilms (Beyenal et al., 2004). For example, our results emphasize the potential of AP and MDD to extract key attributes of biofilm structures, as the presence, location and relative dimension of cells aggregations, biofilm density and distribution of viable and dead cells within biofilms. More specifically the use of these parameters as functions of biofilm height is more informative than their volumetric definition.

Besides the study of the dynamics reveals how different strains follow different paths in their life cycles. The use of 3D and 2D parameters enables the quantification of such differences, not only on the magnitudes of the various events but also in the timing. In fact, though all strains considered seem to follow a similar cycle: attachment, cluster formation, honeycomb-type structure, flat pattern and detachment; durations and magnitudes of the different phases are entirely different among them. These differences clearly determine the physiological properties of the biofilm structures. Although the phases detected in this work are quite similar to those observed in previous works (Mosquera-Fernández et al., 2014; Pilchová et al., 2014); importantly results vary especially in what concerns to the timing. These differences can be due to the use of different surfaces and growth conditions but also to the fact that some strains may skip some phases.

Guilbaud et al., 2015 observed biofilm structures at $48 \mathrm{~h}$ showing mostly honeycomb-like patterns, except for those strains without flagellum for which flat patterns were observed. Pilchová et al., 2014 studied structures at 1, 3 and six days and suggested that some strains could skip the honeycomb-like pattern during the life cycle, evolving to flat patterns. Note however that the temporal resolution used may not allow observing the honeycomb-like as it may appear and disappear at intermediate times. In our analysis, we confirm that the honeycomb-like phase is either too short (less than one day), or it is skipped for some strains. In particular, we do not observe the honeycomb-like pattern for CECT4032 and it is hard to assess whether later stages correspond to flat structures or unstructured populations. In addition, for L1A1 the honeycomb pattern is not as clear as for the case of CECT5873. This can be clearly observed in confocal images Figure 3 as well as Figures S1\& S2 (in supplementary material); but also in the lower MDD 
values obtained for CECT4032 and L1A1 (Figures 6) as compared to CECT5873 for which the honey comb pattern is clear.

Bridier et al., 2010 had previously noted that different L. monocytogenes strains result in biofilms of different heights. Marsh et al., 2003 after imaging the L. monocytogenes biofilms formed by Scott A, V7 and F2365 strains on two different surfaces using scanning electron microscopy (SEM), also observed inter-strain variability in biofilm formation. They argued that the initial attachment step largely conditions biofilm structure. In contrast, what we observe would indicate that distribution at $1 \mathrm{~h}$ does not influence ulterior distributions as also noted by Chae and Schraft, 2000 or Kalmokoff et al., 2001. This becomes apparent when observing the AP and MDD values. All of them are practically the same at sampling time $1 \mathrm{~h}$, but they rapidly evolve to different values. Figure $\mathrm{S} 1$ in the supplementary material confirms this fact, while at time $1 \mathrm{~h}$ cells distribution is quite random in the surface at time $4 \mathrm{~h}$ completely different structures are observed. This lack of influence would indicate that other processes have to be taken into account: cell deposition, motility, and physiology of the cell (growth, nutrient consumption, the production of extracellular polymeric substances).

Our results conclude that L1A1 forms the most stable biofilm: with denser long lasting structures. In general, biofilms are wrinkled, with the highest height of around $50 \mu \mathrm{m}$ and the maximum biovolume around $6 \times 10^{5}$, both values are higher than those observed in CECT5873 and CECT4032 (Figure 4). It should be emphasized that cells located at lower layers are those dying first, indicating that it may be difficult for nutrients to arrive at those lower layers.

CECT5873 presents a faster dynamics. Biofilms achieve the maximum heights of around $35 \mu \mathrm{m}$ at $24 \mathrm{~h}$ (Figure 4A), forming mushroom-type clusters (Figure 3 and 6C-D). This type of clusters is quite unusual in L. monocytogenes biofilms (Rieu et al., 2008). After that cells start dying and detaching giving rise to honeycomb-type structures, which rapidly disappear. One hypothesis to explain this behaviour would be that dead cells contribute to new adhesion. In this respect, recent studies (Bayles, 2007) comment on the role that extracellular DNA from lysed cells could have in intercellular adhesion and biofilm stability. 
CECT4032 seems to keep a constant height around $20 \mu \mathrm{m}$ throughout time (Figure 3). In addition 2D results show the presence of small clusters in some phases. These clusters may be occupied by both live and dead cells as evidenced by 2D image parameters (Figures 5E and 5F).

It is difficult to establish a single reason to explain the differences in the behaviour of these two strains, CECT5873 and CECT4032, with respect to the L1A1. The hypothesis would be that L1A1 is more suited to sustain a biofilm structure on its own. Also the differences in motility of theses strains, reported in a previous study (Mosquera-Fernández et al., 2014), could explain the differences observed at earlier times as also pointed by other authors (Guilbaud et al., 2015). The fact that L1A1 is the less motile could explain that the biofilm is wrinkled instead of forming clusters like the ones observed in CECT4032, the most motile.

\section{CONCLUSION}

This work presented for the first time the numerical spatiotemporal characterization of biofilms formed by three L. monocytogenes strains on polystyrene. CLSM combined with multi-dimensional image analysis tools, PHLIP and BIOFILMDIVER, were used for the purpose of quantifying biofilms maximum thickness, biovolume, areal porosity and maximum diffusion distance.

Results showed the potential of combining quantitative 2D and 3D image analysis to characterize biofilm structure temporal evolution. On the one hand, 3D parameters enable the possibility of comparing volumetric measures, such as biovolume or maximum height. On the contrary, 2D parameters are more significant in what respects to structural features such as the presence and location of clusters, channels, etc. as well as the location and appearance of dead cells. The use of CLSM offers further advantages since it is possible to determine $2 \mathrm{D}$ parameters at different heights.

The approach was used to characterize biofilms formed by three L. monocytogenes strains. The analysis of results showed a common pattern sequence: separate clusters, clusters interconnections, honeycomb-like structures, flat pattern and finally decay and detachment. The time is a critical variable, revealing clear differences in the durations of the different phases for the different strains. Concerning 
specific results, L1A1 leads to the thickest and most stable biofilms. CECT5873 and CECT4032 result in thinner structures with a faster life cycle.

Differences in temporal dynamics emphasize the importance of performing detailed quantitative spatiotemporal studies. In this concern, the quantitative analysis proposed in this work will undoubtedly contribute to, first, identify the physiological state of the biofilms and, second, to the reliable design of disinfection techniques.

\section{Acknowledgements}

Both the Spanish MINECO (ENZYMONO, AGL2010-22212-CO2-02) and the Axencia Galega de Investigación (GAIN) funded this research. M. Mosquera-Fernández acknowledges the financial support from the JAE-CSIC program co-founded by FEDER.

\section{REFERENCES}

Bayles, K.W., 2007. The biological role of death and lysis in biofilm development. Nat Rev Microbiol. 5, $721-726$

Beyenal, H., Donovan, C., Lewandowski, Z., Harkin, G., 2004. Three-dimensional biofilm structure quantification. . J Microbiol Methods. 59, 395-413

Bridier, A., Dubois-Brissonnet, F., Boubetra, A., Thomas, V., Briandet, R., 2010. The biofilm architecture of sixty opportunistic pathogens deciphered using a high throughput CLSM method. J Microbiol Methods. 82, 64-70.

Carpentier, B., Cerf, O., 2011. Review-Persistance of Listeria monocytogenes in foof industry equipement and premises. Int J Food Microbiol.145, 1-8.

Chae, M.S., Schraft, H., 2000. Comparative evaluation of adhesion and biofilm formation of different Listeria monocytogenes strains. Int J Food Microbiol. 62, 103-111. 
Characklis, W.G., Mashall, K.C., 1990. Biofilms, ed. John Wiley, New York.

Costerton, J., Cheng, K., Geesey, T., Nickel, J., Dasgupta, M., Marrie, T., 1987. Bacterial Biofilms Nature and Disease. Annu Rev Microbiol. 41, 435-464

Danielsson, P., 1980. Euclidean Distance Mapping. Comput Vision Graph. 14, 227-248.

Djordjevic, D., Wiedmann, M., McLandsborough, L.A., 2002. Microtiter plate assay for assessment of Listeria monocytogenes biofilm formation. Appl Environ Microbiol. 68, 2950-2958.

European Food Safety Authority (EFSA) and European Centre for Disease Prevention and Control (ECDC) 2015. The European Union summary report on trends and sources of zoonoses, zoonotic agents and food-borne outbreaks in 2013. EFSA Journal. 13, 1-3991.

Fux, C.A., Costerton, J.W., Stewart, P.S., Stoodley, P., 2005. Survival strategies of infectious biofilms. Trends Microbiol. 13, 34-40.

Guilbaud, M., Piveteau, P., Desvaux, M., Brisse, S., Briandet, R., 2015. Exploring the diversity of Listeria monocytogenes biofilm architecture by high-throughput Confocal Laser Scanning Microscopy and de Predominance of Honeycomb-like morphotype. Appl Environ Microbiol. 81, 1813-1819.

Heydorn, A., Nielses, A.T., Hentzer, M., Sternberg, C., Givskov, M., Ersboll, B.K., Molin, S., 2000. Quantification of biofilm structure by novel computer program COMSTAT. Microbiology+. 146, 23952407.

Hoiby, N., Bjarnsholt, T., Givsko, M., Molin, S., Ciofu, O., 2010. Antibiotic resistance of bacterial biofilms. Int J Antimicrob Agents. 35, 322-332. 
Kalmokoff, M.L., Austin, J.W., Wan, X.S., Sanders, G., Banerjee, S., Farber, J.M., 2001. Adsorption, attachment and biofilm formation among isolates of Listeria monocytogenes using model conditions. J Appl Microbiol. 91, 725-734.

Kuehn, M., Hausner, M., Bungartz, H.J., Wagner, M., Wilderer, P.A., Wuertz, S., 1998. Automated confocal laser scanning microscopy and semiautomatic image processing for analysis of biofilms. Appl Environ Microbiol. 64, 4115-4127.

Ludén, J.M., Miettinen, M.K., Autio, T.J., Korkeala, H.J., 2000. Persistent Listeria monocytogenes strains show enhanced adherence to food contact surface after short contact time. J Food Protection. 9, 12041207.

Marsh, E.J., Luo, H., Wang, H., 2003. A three-tiered approach to differentiate Listeria monocytogenes biofilm-forming abilities. FEMS Microbiol Lett. 228, 203-210.

Mosquera-Fernández, M., Rodríguez-López, P., Cabo, M.L., Balsa-Canto, E., 2014. Numerical spatiotemporal characterization of Listeria monocytogenes biofilms. Int J Food Microbiol. 182/183, 26-36.

Mueller, L.N., de Brouwer, J.F., Almeida, J.S., Stal, L.J., Xavier, J.B., 2006. Analysis of a marine phototrophic biofilm by confocal laser scanning microscopy using the new image quantification software PHLIP. BioMedCentral Ecology. 6, 1-15.

Pan, Y., Breidt Jr, F., Kathariou, S., 2006. Resistance of Listeria monocytogenes biofilms to sanitizing agents in a simulated food-processing environment. Appl Environ Microb. 72, 7711-7717.

Pilchová, T., Hernould, M., Prévost, H., Demnerová, K., Pazlarová, J., Tresse, O., 2014. Influence of food processing environments on structure initiation of static biofilm of Listeria monocytogenes. Food Control. $35,366-372$.

Rieu, A., Briandet, R., Habimana, O., Garmyn, D., Guzzo, J., Piveteau, P., 2008. Listeria monocytogenes EGD-e biofilms: no mushrooms but a network of knitted chains. Appl Environ Microbiol. 74, 4491-4497. 
Robbins, J.B., Fisher, C.W., Moltz, A.G. and Martin, S.E., 2005. Elimination of Listeria monocytogenes biofilms by ozone, chlorine, and hydrogen peroxide. J Food Protection. 68, 494-498.

Somers, E.B., Wong, A.C., 2004. Efficacy of two cleaning and sanitizing combinations on Listeria monocytogenes biofilms formed at low temperature on a variety of materials in the presence of ready-toeat meat residue. J Food Protection. 67, 2218-2229.

Stewart, P.S., 2003. Diffusion in Biofilms. J Bacteriol. 185, 1485-1491.

Stoodley, P., Boyle, J.D., DeBeer, D., Lappin-Scott, H.M., 1999. Evolving perspectives of biofilm structures. Biofouling. 14, 75-90.

Wilks, S.A., Michels, H.T., Keevil, C.W., 2006. Survival of Listeria monocytogenes Scott A on metal surfaces: Implications for cross-contamination. Int J Food Microbiol. 111, 93-98.

Yang, X., Beyenal, H., Harkin, G., Lewandowski, Z., 2000. Quantifying biofilms structure using image analysis. J Microbiol Methods. 39, 109-119.

\section{LIST OF FIGURES}



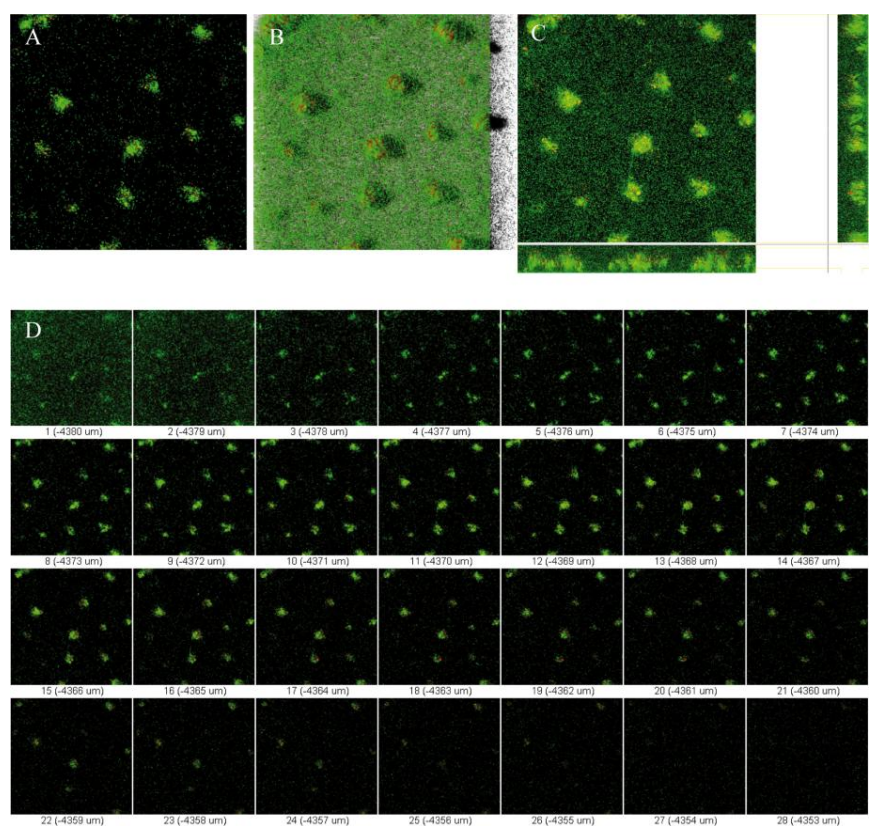

Fig. 1: IMARIS view modes. A: Slice, B: Blend, C: Section, D: Gallery

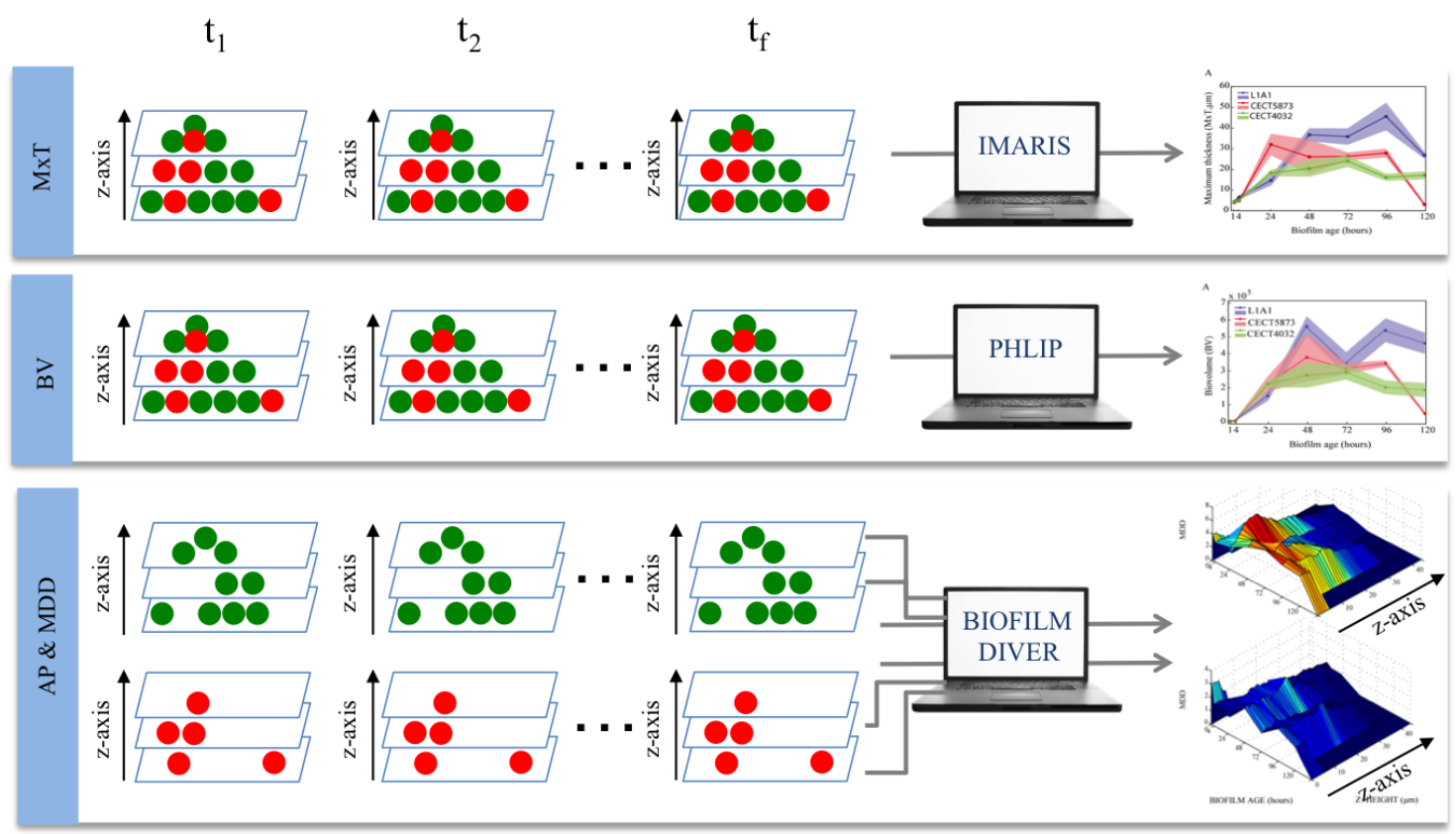

Fig. 2: Image quantitative analysis steps 

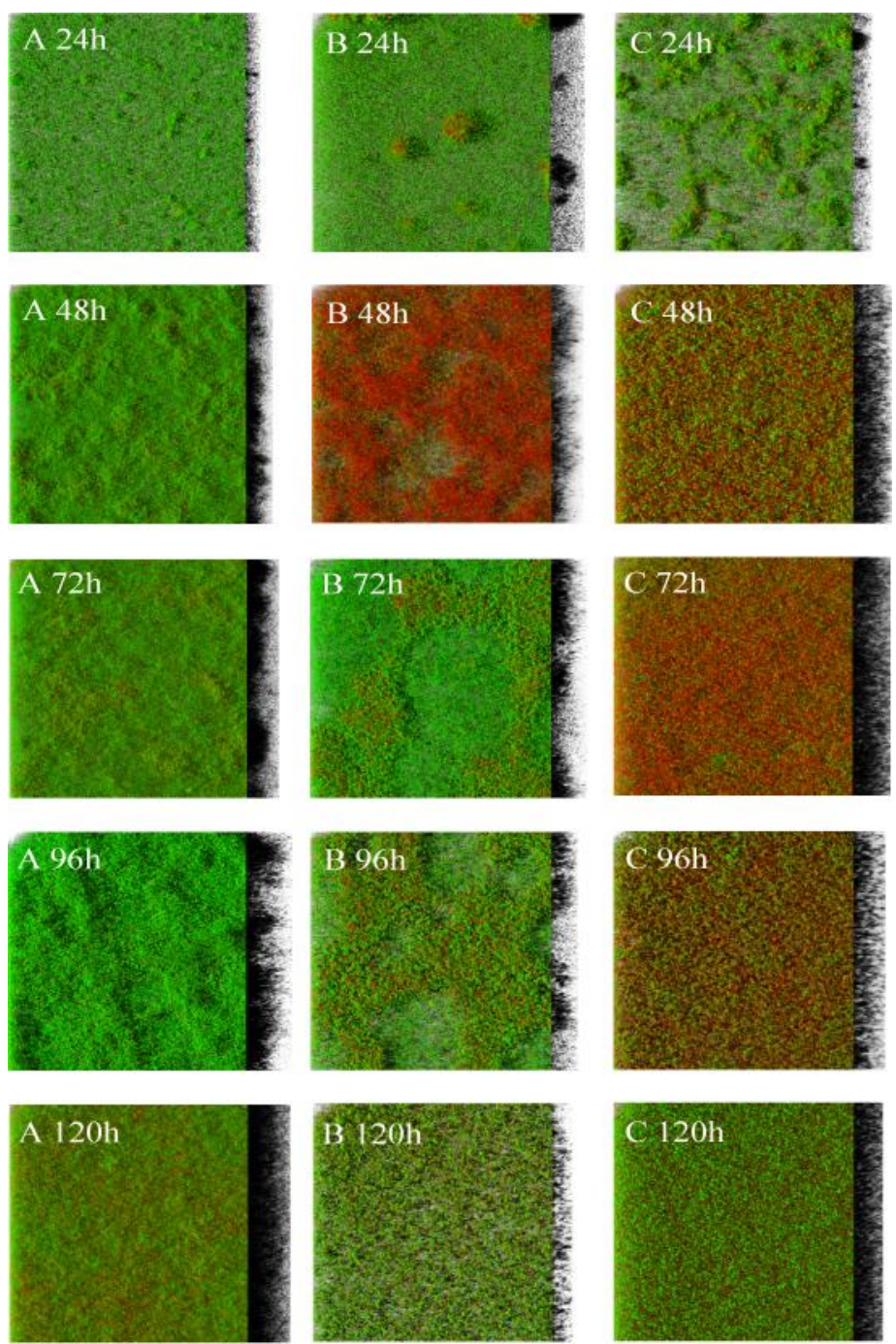

Fig. 3: Biofilms states at five sampling times of A: L1A1, B: CECT 5873 and C: CETC 4032 strains, from $24 \mathrm{~h}$ to $120 \mathrm{~h}$ as shown in IMARIS blend view mode.
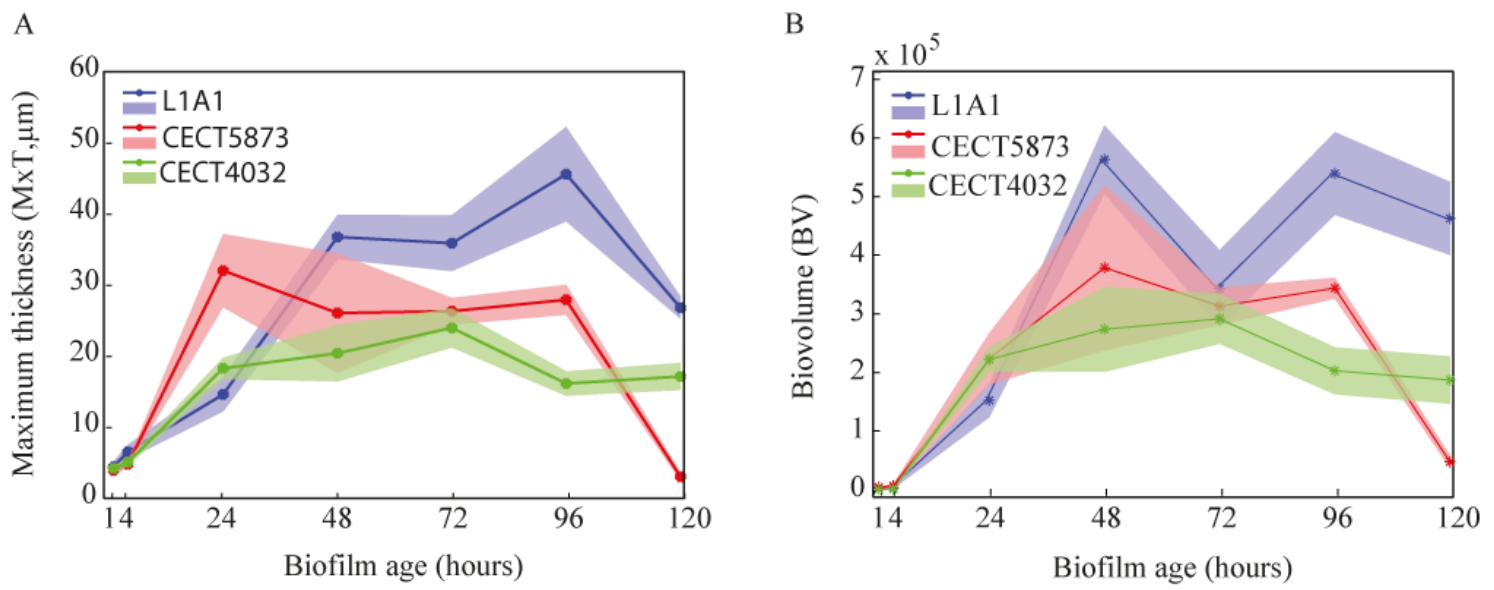
Fig. 4: A. Maximum Thickness (MxT) and B. Biovolume (BV) determinations for L1.A1 biofilms (blue), CECT5873 biofilms (red) and CECT4032 biofilms (green). Dots correspond to mean value; dark shades correspond to $75 \%$ confidence region. Independent figures for each strain are available at Figure 3 and 4 of supplementary material.

A

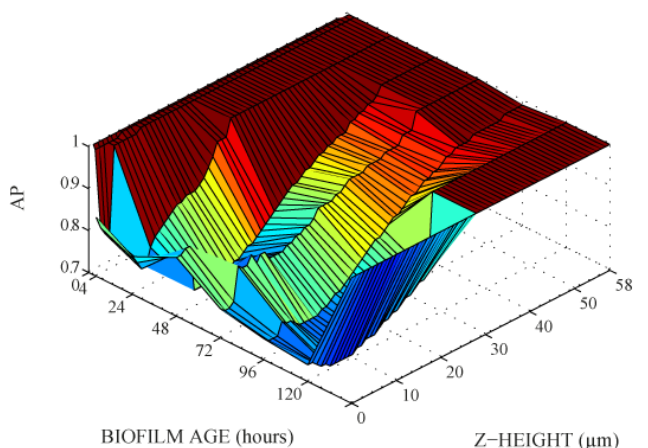

B

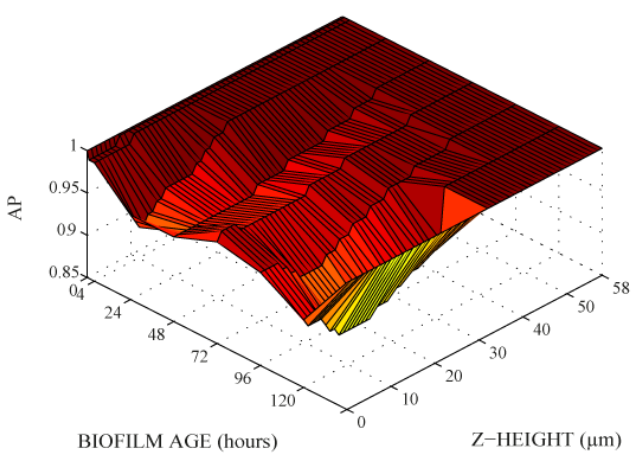

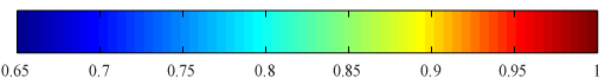

C AREAL POROSITY (GCh)

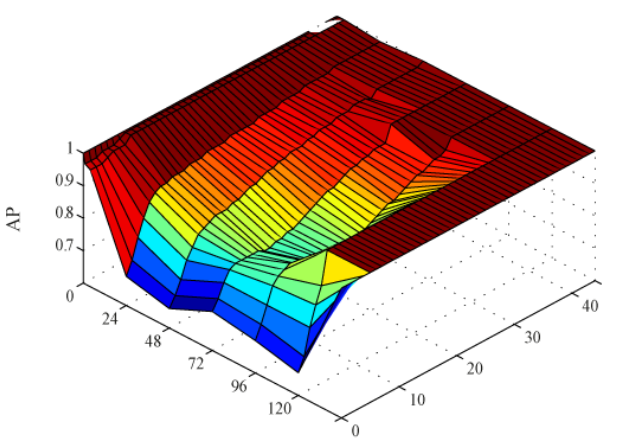

D BIOFILM AGE (hours) Z-HEIGHT $(\mu \mathrm{m})$

AREAL POROSITY (RCh)

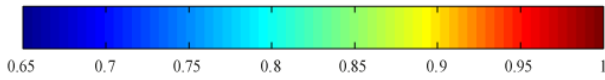

E AREAL POROSITY (GCh)

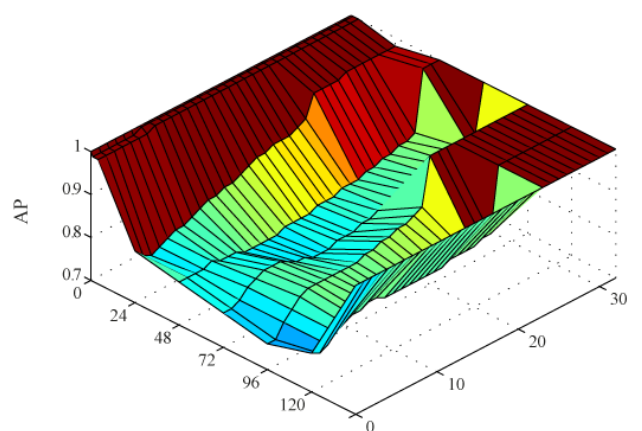

F

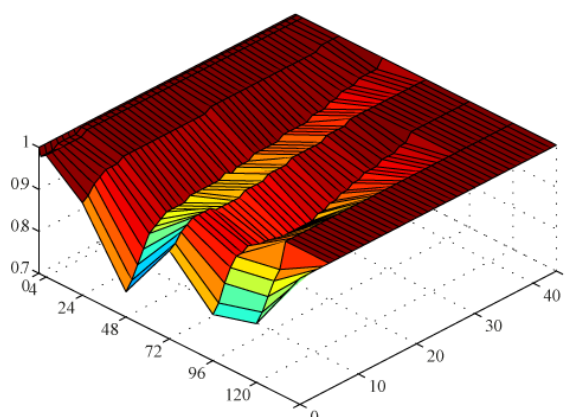

BIOFILM AGE (hours)

Z-HEIGHT ( $\mu \mathrm{m})$ BIOFILM AGE (hours)

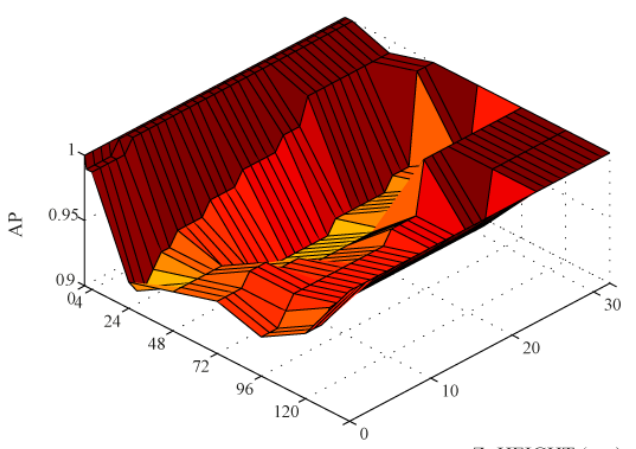

BIOFILM AGE (hours)

Z-HEIGHT $(\mu \mathrm{m})$

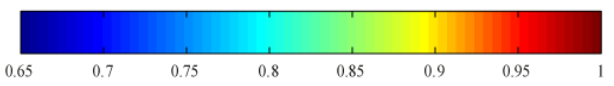

Fig. 5: AP as computed by BIFILMDIVER. A, B: GCh and RCh for L1A1; C-D: GCh and RCh for CECT 5873; E-F: GCh and RCh for $\mathrm{CECT} 4032$. $\mathrm{AP}=1$ (dark red colors) indicates absence of cells. 


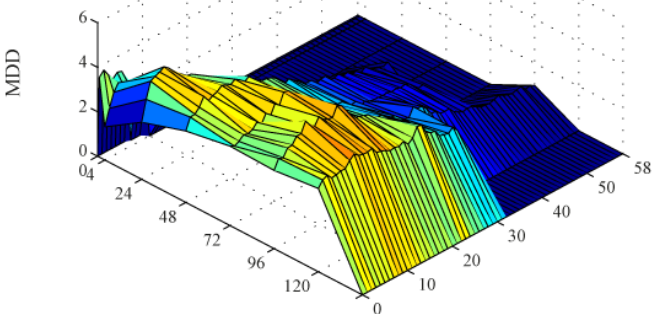

BIOFILM AGE (hours)

Z-HEIGHT $(\mu \mathrm{m})$

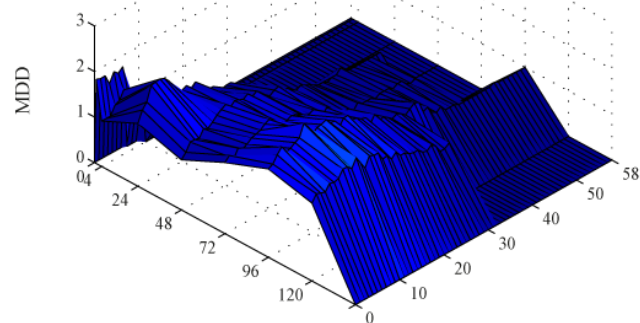

BIOFILM AGE (hours)

Z-HEIGHT $(\mu \mathrm{m})$

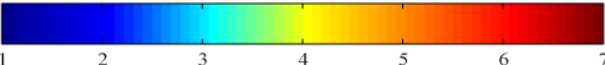

C

MAXIMUM DIFFUSION DISTANCE (GCh)

D

MAXIMUM DIFFUSION DISTANCE (RCh)
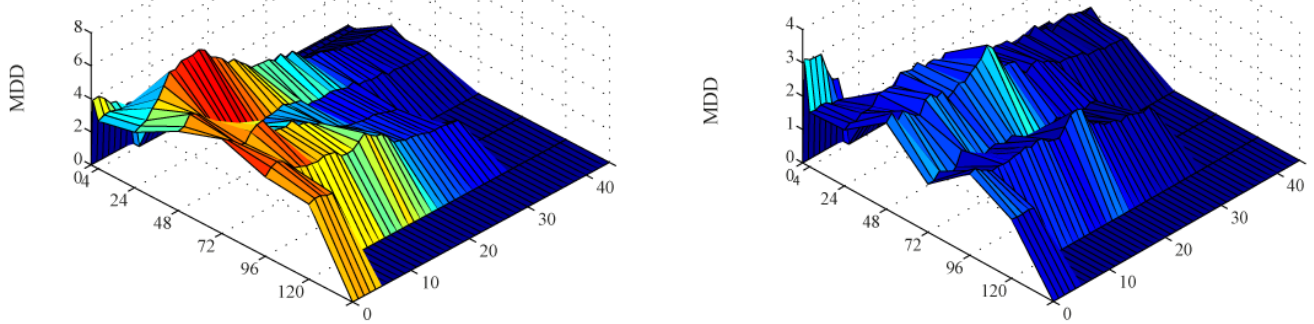

BIOFILM AGE (hours)

Z-HEIGHT $(\mu \mathrm{m})$

BIOFILM AGE (hours)

Z-HEIGHT $(\mu \mathrm{m})$

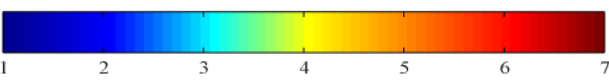

E
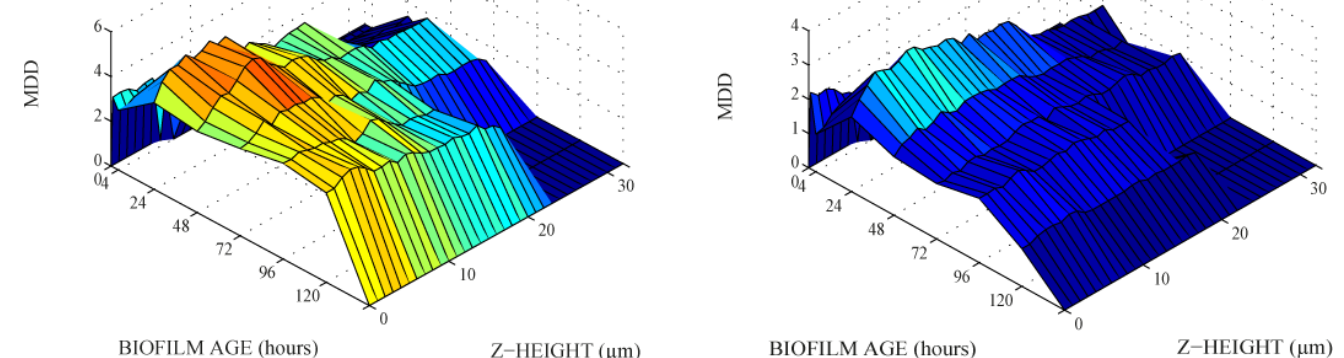

BIOFILM AGE (hours)

Z-HEIGHT $(\mu \mathrm{m})$

BIOFILM AGE (hours)

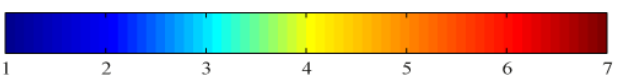

Fig. 6: MDD as computed by BIFILMDIVER. A, B: GCh and RCh for L1A1; C-D: GCh and RCh for CECT 5873; E-F: GCh and RCh for CECT4032. 


\section{HIGHLIGTHS}

- $2 \mathrm{D}$ and 3D quantitative image analyses allow characterizing the dynamics of biofilms.

- 2D parameters identify structures and appearance and location of dead cells.

- 3D parameters evaluate thickness and biovolume facilitating life cycle studies.

- Three Listeria monocytogenes strains follow common patterns during life cycle.

- Magnitude and duration of patterns are different: the role of time is critical. 